\title{
Finding fulfillment in a psychiatry clinical teaching role
}

Alexandra L. Dodd, MD

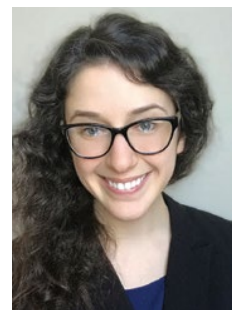

Dr. Dodd is Chief Resident, PGY-4, Department of Psychiatry, Prisma Health-University of South Carolina School of Medicine, Greenville, South Carolina.

Disclosure

The author reports no financial relationships with any companies whose products are mentioned in this article, or with manufacturers of competing products.

doi: $10.12788 /$ cp.0096

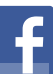

Discuss this article at www.facebook.com/ MDedgePsychiatry
$\mathrm{O}$ n my third day as a PGY-4 junior attending on the inpatient psychiatric ward, 2 new PGY-1 residents, 2 medical students, and I stood in the wee hours of the morning, preparing to meet with our attending to begin rounds. I took the opportunity to discuss potential antipsychotic selection for one of our patients. I questioned the students to gauge their level of knowledge on antipsychotics in general, and did some "thinking out loud" about what our possible options could be. We discussed which antipsychotics are considered "weight-neutral" and which ones require caloric intake for adequate absorption. We discussed what other laboratory tests we should consider upon initiating the hypothetical medication. While discussing these things, I was suddenly taken aback to see that every member of my team was diligently taking notes and hanging on my every word!

\section{Lessons from my teaching experiences}

Taking on the role of junior attending has made me reflect on a few things about the transition that I will undergo at the end of this year, from resident to attending. First, teaching makes me keen to really sharpen my own knowledge, so that I can provide accurate information with confidence and ease. Making valid clinical decisions is a basic attending skill, but eloquently explaining clinical decisions to trainees with varying levels of background knowledge is a unique teaching attending necessity.

Second, I had this amazing feeling of helping patients beyond those currently in my care, since disseminating useful clinical information will allow trainees to better prepare to treat their own patients later. Random hypothetical situations presented by my attendings through the years may have seemed tangentially related to rounds at the time, but were meant to prepare me for actual future decisions (for example, "What would you change if this patient's renal impairment were more severe?"). These teaching moments strengthen problem-solving skills and help us get as much benefit as possible from each case. The service to future patients extends to students who aren't pursuing careers in psychiatry, because the skills they learn during a psychiatry rotation will help them connect with patients in any setting.

Third, I realized that teaching has the power to actively shape the future of medicine. What my attendings have taught me through the years is echoed and amplified in my teaching, and supplemented with my own readings and practice patterns.

Fourth, I noted what a privilege it is to be in a field with such attentive and eager trainees; as teachers in medicine, we truly get to work with the cream of the crop,

continued on page 45

LET YOUR VOICE BE HEARD

CURRENT PsyChIATRY invites psychiatry residents to share their views on professional or clinical topics for publication in Residents' Voices. E-mail jbauer@mdedge.com for author guidelines. 
Psychiatry clinical teaching role continued from page 38

which is a joy and a great responsibility. Working with such highly motivated and attentive students can be intimidating, but as I realized later in the morning, when asked about the complete indications for gabapentin, I realized I'm comfortable saying, "I don't know, let's look it up together!"

My fifth and final realization from this exciting teaching experience was that as an attending, I will need to help manage the wellness and growth of my trainees. Attendings must strike a balance between pushing learners to gain mastery while protecting them from excessive stress. I am so grateful for the perceptiveness of my attendings and their ability to adapt to the demands of a clinical environment while maintaining a strong focus on teaching. I have often told PGY-1 residents, when they face feelings of inadequacy for early mistakes, "You have 4 whole years to learn how to do this job!"

These are the moments that make me appreciate the fulfillment that can come from teaching residents and medical students, and really put into perspective how far I've come as a trainee. Not long ago, I was one of those medical students scribbling notes while my attending effortlessly spouted medical knowledge, and I was worried I'd never learn the difference between clonidine and clozapine.

\section{Clinical Point}

Teaching makes me keen to really sharpen my own knowledge, so that I can provide accurate information with confidence and ease 